\title{
History-dependent synchronization in the Burridge-Knopoff model
}

\author{
J. Szkutnik, B. Kawecka-Magiera, K. Kułakowski* \\ Faculty of Physics and Nuclear Techniques, AGH University of Science and Technology \\ al. Mickiewicza 30, 30059 Kraków, Poland. \\ *Corresponding author.E-mail: kulakowski@novell.ftj.agh.edu.pl
}

October 30, 2018

A three-blocks Burridge-Knopoff model is investigated. The dimensionless velocity-dependent friction force $F(v) \propto(1+a v)^{-1}$ is linearized around $a=0$. In this way, the model is transformed into a six-dimensional mapping $\mathbf{x}\left(t_{n}\right) \rightarrow \mathbf{x}\left(t_{n+1}\right)$, where $t_{n}$ are time moments when a block starts to move or stops. Between these moments, the equations of motion are integrable. For $a<0.1$, the motion is quasiperiodic or periodic, depending on the initial conditions. For the periodic solution, we observe a synchronization of the motion of the lateral blocks. For $a>0.1$, the motion becomes chaotic. These results are true for the linearized mapping, linearized numerical and non-linearized numerical solutions.

Keywords: nonlinear dynamics, stick-slip, coupled maps

]

\section{Introduction}

We consider the Burridge-Knopoff model (BKM) of three blocks 11. This is a simple chain of blocks connected with springs, with additional springs which drive each block with a constant velocity $V$. The blocks move on a surface with friction. The model belongs to a rich branch of many-body dissipative systems: the train model [2], the Feder and Feder model [3] and the OlamiFeder-Christensen model 4]. There are also some connection from this branch to the Frenkel-Kontorova model [5]. At the root of the whole Tree of many-body nonlinear systems there is the fameous Fermi-Pasta-Ulam model [ 6 . There is also a conservative branch, with the Toda chain [7], ding-dong [8] and ding-a-ling 9] models and presumably some others as leaves.

Most characteristical effect displayed by the dissipative branch of the Tree is the stick-slip dynamics: the blocks move or stop at various time moments, periodically for one block and perhaps chaotically for two or more blocks. Recent works of Persson 13, 14] allow to treat BKM as a generic model of two surfaces, 


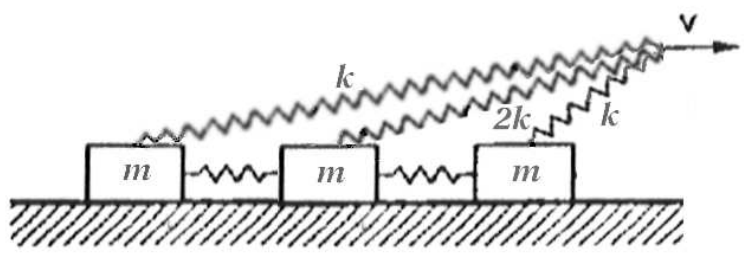

Figure 1: The Burridge-Knopoff system considered in this work. Three equal masses $m$ are drawn along a rough platform with constant velocity $V$. The masses are connected with springs of strength $k$. Similar springs are used to attach them to the driving mechanism.

one sliding on another with friction. On the other hand, the stick-slip dynamics has been observed experimentally in nanoscopic systems [15. In particular, this kind of motion turned out to be relevant for the behaviour of tips of the scanning probe microscopes.

The origin of the stick-slip dynamics has been often discussed in terms of the state and the rate dependent model [16, 17, 18]. There, the character of the velocity dependence of the friction force, $F(v)$, is considered to be crucial. On the other hand, as it was demonstrated in [2], the stability of the uniform motion depends only on the sign of the drivative $F^{\prime}(v)$.

Usually, the discussion of all the above listed models concentrates on two topics: their application to theory of earthquakes (which are at the origin of the problem [10]) and the effects of self-organized criticality [11. Both topics overlap at the Richter-Gutenberg law, which is an example of a scaling relation which has been observed out of laboratory [12]. Other effects under discussion within the above models are chaos and synchronization [1. All these topics are often considered for large systems, despite the fact that even small systems of this kind are not entirely known. The aim of this work is to show some unexpected properties of the Burridge-Knopoff system of three blocks.

In its original version [1] the BKM is nonlinear because of two reasons. First is that the friction force $F$ acting on a block depends on its velocity $v$ in a nonlinear way, i.e. $F=F(0) /(1+c v)$. Second is that the equations of motion are nondifferentiable at time moments when a block starts to move or stops. In our previous paper [19] we have demonstrated that for two blocks and small values of the parameter $c$, the first cause of nonlinearity is not relevant. Namely, we substituted the nonlinear function $(1+c v)^{-1}$ by its linear approximation $1-c v$. This step is justified for small values of $c$, i. e. for strong spring constant $k$, large mass $m$ and low friction force which only weakly depends on the velocity $v$. On the other hand, this step allows to integrate the equations of motion analytically. It is useful to introduce here a new variable $a=c F(0) / \sqrt{\mathrm{km}}$. It has been shown in Ref. [19] that for $a<0.3$, the results of the original and linearized versions are practically the same. 


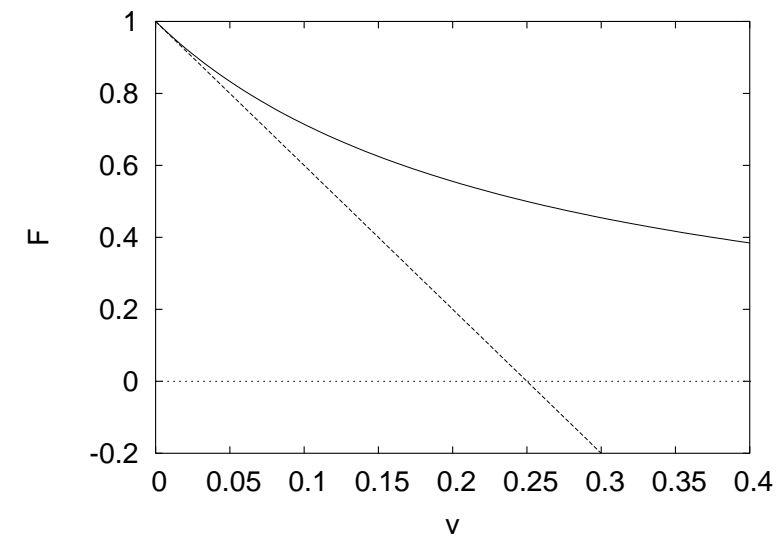

Figure 2: Velocity dependent function of friction force $F(v)$. Solid line for nonlinear form, dashed line for linearized form. Dotted line is a guide for eye.

The goal of the present work is twofold. First observation is that the above mentioned linearization does not change the results of BKM for three blocks, when the parameter $a$ is less than about 0.1. Second observation is that the character of the motion can depend on the history of the system. Near $a=0.1$ we observe a transition from periodic or quasi-periodic behaviour for small $a$ to chaos for larger $a$. The character of the motion for $a<0.1$ does depend on the initial conditions. Changing only the initial position of one of the blocks, we switch from a quasi-periodic and non-synchronized motion to a periodic evolution with the synchronization of two lateral blocks. This switching from one kind of motion to another is the same for the linearized motion and for the fully nonlinear formulation. In the latter case, the only solution is numerical. Here we use the Runge-Kutta method of fourth order.

The paper is organized as follows. In the next section we describe the linearization procedure. The outcoming equations are piecewisely linear and they are solved analytically. The solution is valid except the time moments, when the number of moving blocks varies. These time moments are found numerically. In Section III we show the results obtained within the linearized model and within its initial formulation - the latter is worked out numerically. The last section is devoted to conclusions. 


\section{The linearization}

The starting point for the linearization procedure is the set of three equations of motion for three blocks

$$
\begin{aligned}
& m \ddot{X}_{1}=k\left(X_{2}-2 X_{1}+V t\right)-F\left(c \dot{X}_{1}\right) \\
& m \ddot{X}_{2}=k\left(X_{1}-4 X_{2}+X_{3}+2 V t\right)-F\left(c \dot{X}_{2}\right) \\
& m \ddot{X}_{3}=k\left(X_{2}-2 X_{3}+V t\right)-F\left(c \dot{X}_{3}\right)
\end{aligned}
$$

where $F(c \dot{X})=F(0) /(1+c \dot{X}), X_{i}$ is the position of $i$-th block, $V t$ is the position of the driving mechanism. The driving spring constants are chosen to be $k, 2 k$ and $k$ for the first, second and third block respectively, and the spring constants of the springs between the blocks are all equal to $k$. Equation for $i$-th block is valid as long as it moves. When the block stops, the condition of moving it again is that the total spring force acting on the block must be greater than $F(0)$.

If according to [1] we change spatial and time units as follows: $\tau=\omega t, U_{j}=$ $k X_{j} / F(0), \omega^{2}=k / m$ we can write the equations of motion in dimensionless form:

$$
\begin{aligned}
& \ddot{U}_{1}=U_{2}-2 U_{1}+v \tau-\Phi\left(a \dot{U}_{1}\right) \\
& \ddot{U}_{2}=U_{1}-4 U_{2}+U_{3}+2 v \tau-\Phi\left(a \dot{U}_{2}\right) \\
& \ddot{U}_{3}=U_{2}-2 U_{3}+v \tau-\Phi\left(a \dot{U}_{3}\right)
\end{aligned}
$$

where $v=V \sqrt{k m} / F(0)$ and $a=c F(0) / \sqrt{k m}$. The friction force has now the following shape: $\Phi(a \dot{U})=1 /(1+a \dot{U})$.

For the linearized equations, the only modification is that the friction force $\Phi(a \dot{U})=1-a \dot{U}$. This approximation is justified as long as the constant $a$ is small. Additionally, we have to check whether the friction force changes sign this would be a harmful artifact. The profit is that the equations of motion are analytically integrable: the functions have this shape:

$$
U_{n}=A \exp (\alpha \tau)+B \exp (\beta \tau)+C \exp (\gamma \tau)+D \tau+E
$$

We need to solve a new set of equations whenever one of the block starts to move or stops. This time moments are roots of function (3) and they are to be found numerically.

\section{Results}

In Figs. 3a and 3b we show the time averages of the distance $J(\tau)$ in the phase space between the first and third block. This distance is defined as

$$
J(\tau)=\sqrt{\left(\dot{U}_{3}(\tau)-\dot{U}_{1}(\tau)\right)^{2}+\left(U_{3}(\tau)-U_{1}(\tau)\right)^{2}}
$$



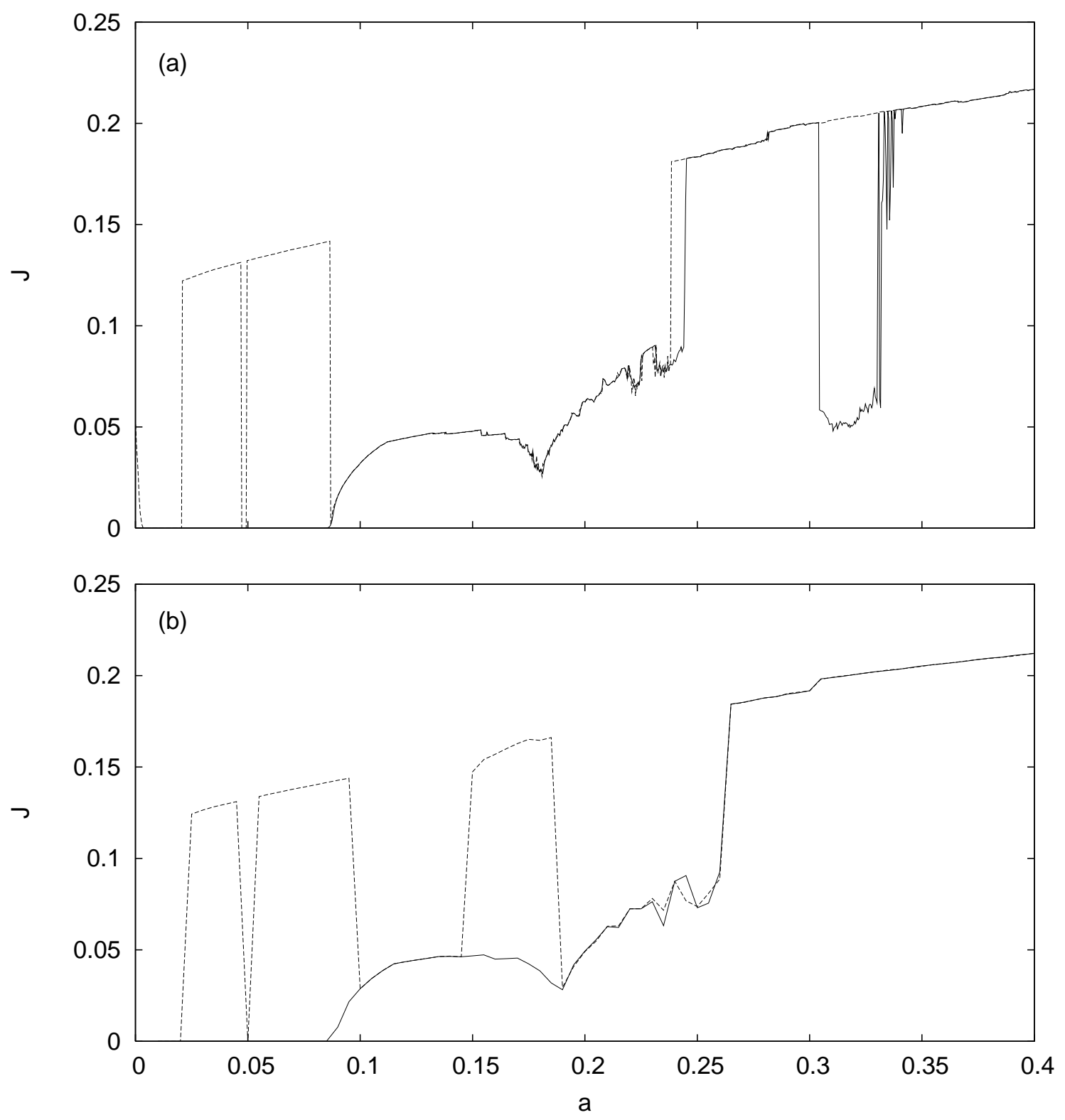

Figure 3: The Euclidean distance $J$ in phase space beetween blocks 1 and 3 as function of $a$. The solid line is for $U_{1}(\tau=0)=+0.989$ in the initial state, and the dotted line - for $U_{1}(\tau=0)=-0.989$ in the initial state. (a) for linearized equation of motion (analytical solution) (b) for nonlinearized equation of motion (numerical solution). 

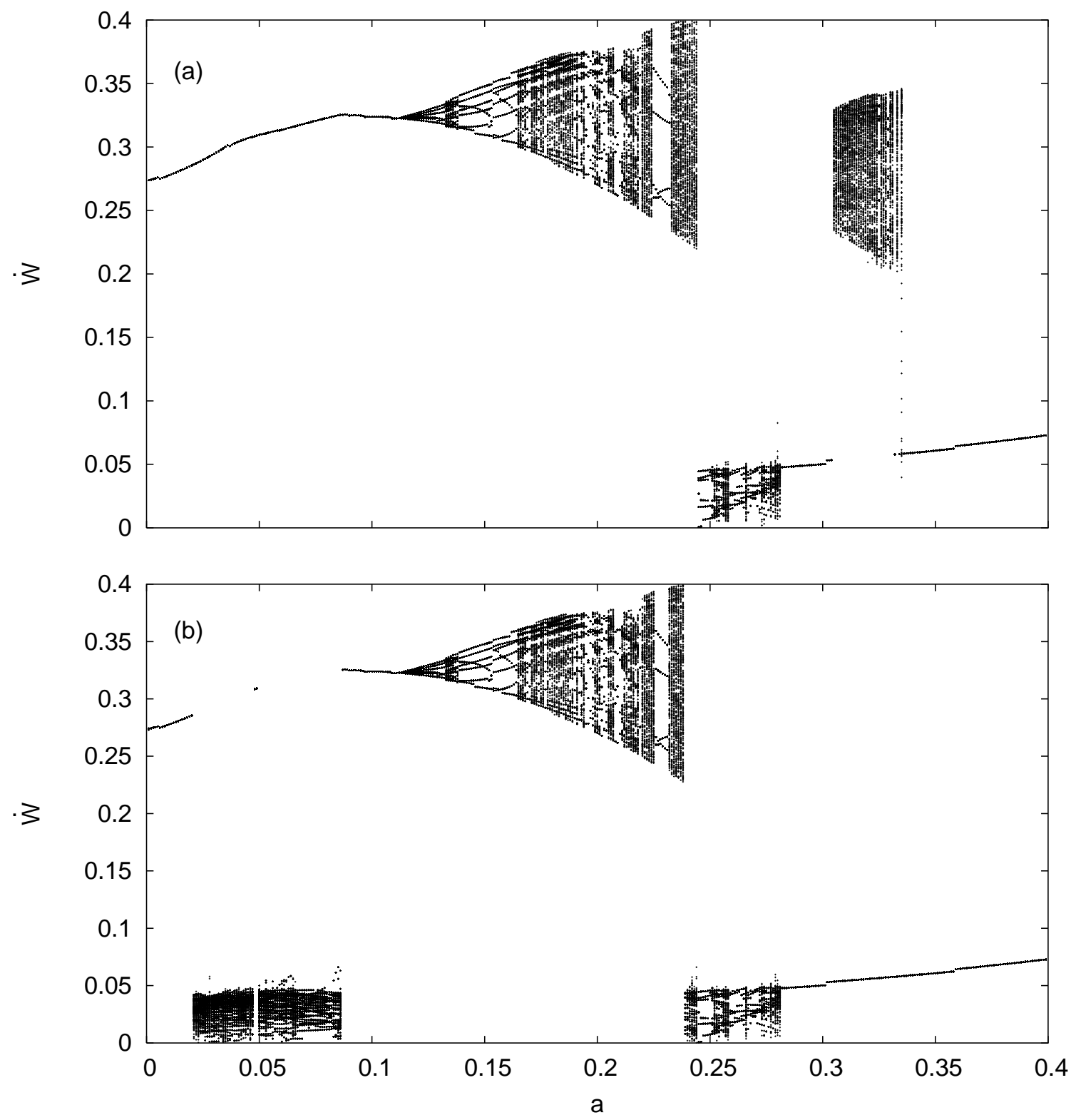

Figure 4: The bifurcation diagram of $\dot{W}$ in the Poincaré section in which $W=0$ (a) for $U_{1}(\tau=0)=-0.989$ in the initial state (b) for $U_{1}(\tau=0)=+0.989$ in the initial state. 
and its time average is performed over a time $\Delta \tau=7 \times 10^{3}$ after a transient of $3 \times 10^{3}$. In the numerical calculations, each time unit is $10^{6}$ time steps. In both analytical and numerical calculations, one stick-slip cycle is about two or three time units. A comparison of the Figs. 3a and $1 \mathrm{~b}$ shows that there is a close correspondence between the results for the initial nonlinear formulation and the linearized one. In both cases, the synchronization appears for the parameter $a$ between 0.005 and 0.08 . In both cases, this synchronization does depend on the initial conditions in the same way. When the initial position of the first block $U_{1}(\tau=0)=-0.989$, i.e. it is close to the position for the stationary state, the motion is synchronized and $\langle J(\tau)\rangle=0$. (Here, $\langle\ldots\rangle$ is the time average.) This is so despite the fact that the stationary motion is unstable. However, for $U_{1}(\tau=0)=+0.989$, the motion is not synchronized and $\langle J(\tau)\rangle$ is positive.

We note that the unstable character of the stationary motion can be proved in the same way as it was done for two blocks by Vieira [2. As long as the friction force decreases with velocity, some eigenvalues of the Jacobian are always positive. The comparison of the Figs. $3 \mathrm{a}$ and $3 \mathrm{~b}$ allows to believe that the linearization procedure does not alter the results for $a<0.1$. Other results presented in Figs. 4-7 are obtained for the linearized equations of motion and for the parameter $a=0.08$. We compare the results for the two above values of the initial position of the first block. The initial positions of the other blocks are $U_{2}(\tau=0)=-0.993$ and $U_{3}(\tau=0)=-0.991$. The initial velocities are $\dot{U}_{1}(\tau=0)=0.099, \dot{U}_{2}(\tau=0)=0.1012$ and $\dot{U}_{3}(\tau=0)=0.1023$. The initial values of the velocities are very close to the driving velocity $v$, which is set as 0.1 .

To construct the bifurcation diagrams, we use the sum of positions of the blocks with respect to the equilibrium positions:

$$
W=U_{1}-U_{1}^{e}+U_{2}-U_{2}^{e}+U_{3}-U_{3}^{e}
$$

We denote $U_{1}^{e}=v \tau-5(1-a v) / 6, U_{2}^{e}=v \tau-2(1-a v) / 3$ and $U_{3}^{e}=v \tau-5(1-a v) / 6$ the unstable point of equilibrium, which is found by taking $\ddot{U}_{n}=0$ and $\dot{U}_{n}=v$ in Eq. (2). The stability of this solution can be calculated as in 2. In Figs. $4 \mathrm{a}$ and $4 \mathrm{~b}$ we show the bifurcation diagrams for the synchronized and nonsynchronized motion. Subsequent points mean the Poincaré surface section of $\dot{W}$ at $W=0$. In the former case (Fig. $4 \mathrm{a}, U_{1}(\tau=0)=-0.989$ ) and below $a=0.1$ we see a continuous line which means one fixed point of the mapping. This means that the whole motion is periodic. In the latter case (Fig. $4 \mathrm{~b}, U_{1}(\tau=0)=+0.989$ ), we see that below $a=0.1$ the above line disappears. Instead, we observe a cloud of points for lower values of the velocity. This means the quasiperiodic or the chaotic motion. To check it, we have calculated the maximal Lyapunov exponent and we have found it to be close to zero.

In Figs. 5a and $5 \mathrm{~b}$ we show the time dependence of the distance $J(\tau)$ between the lateral blocks in the two above cases. In the synchronized case (Fig.5a) $J(\tau)$ tends to zero as soon as for $\tau=4000$, whereas for the nonsynchronized case $J(\tau)$ displays stationary fluctuations around 0.14. Here again, the whole difference is the initial position of the first block. Finally, in Figs. 6 and 7 we show the character of the synchronized and non-synchronized motion. In the former case 

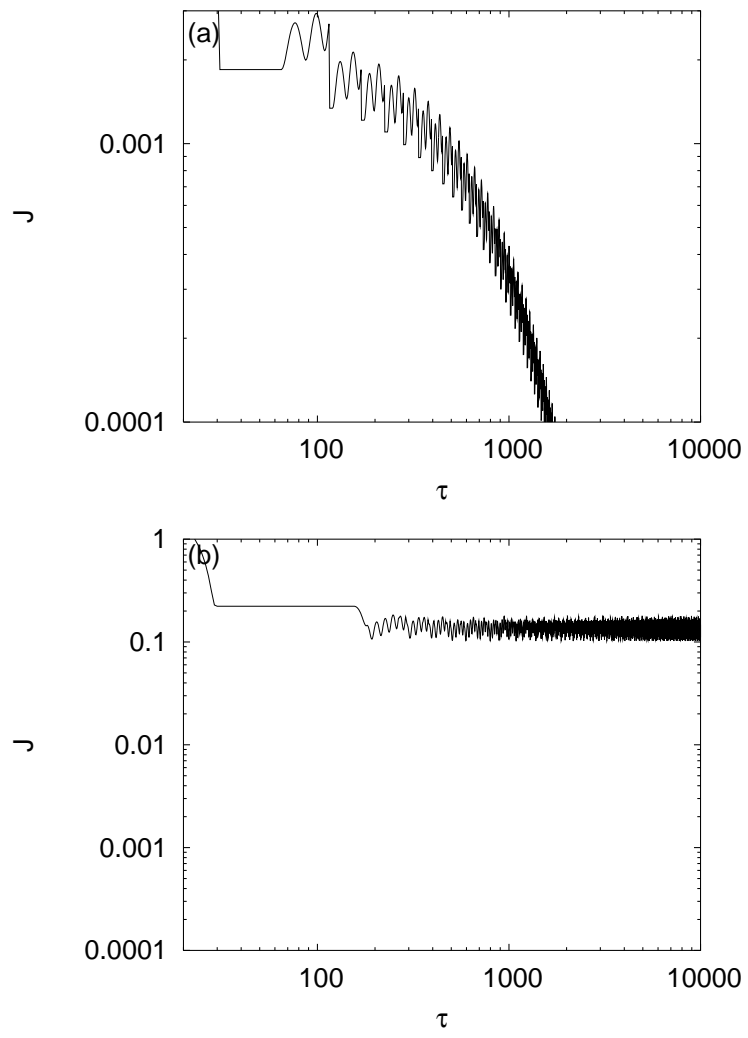

Figure 5: The instantaneous distance in the phase space beetween the lateral blocks (a) for initial position of the 1st block $U_{1}(\tau=0)=-0.989$, (b) for initial position of the 1st block $U_{1}(\tau=0)=+0.989$.

(Fig. 6), the positions and the velocities of the block are periodic functions of time. In the latter case (Fig. 7), the motion is aperiodic. Moreover, the velocities of first and third block seem to be in some kind of antiphase: when one increases, another decreases. Both oscillate with the frequency more or less two times smaller, than the middle block.

As we have seen in Figs. 6a and 7a, the stick-slip effect is present in both cases of periodic and aperiodic motion. The plots of velocities vs. time touch the value zero for each of the three blocks. It seems that some sticks are unavoidable. This conclusion is supported by the fact, that the analytical solution for the linearized mapping contains the term $\exp \left[-F^{\prime}(v) t\right]$, where $F(v)$ is the velocity dependence of the friction force. Therefore, if only the derivative $F^{\prime}(v)$ is negative, the amplitude of the velocity oscillations increases with time and $v$ must reach the zero value. 

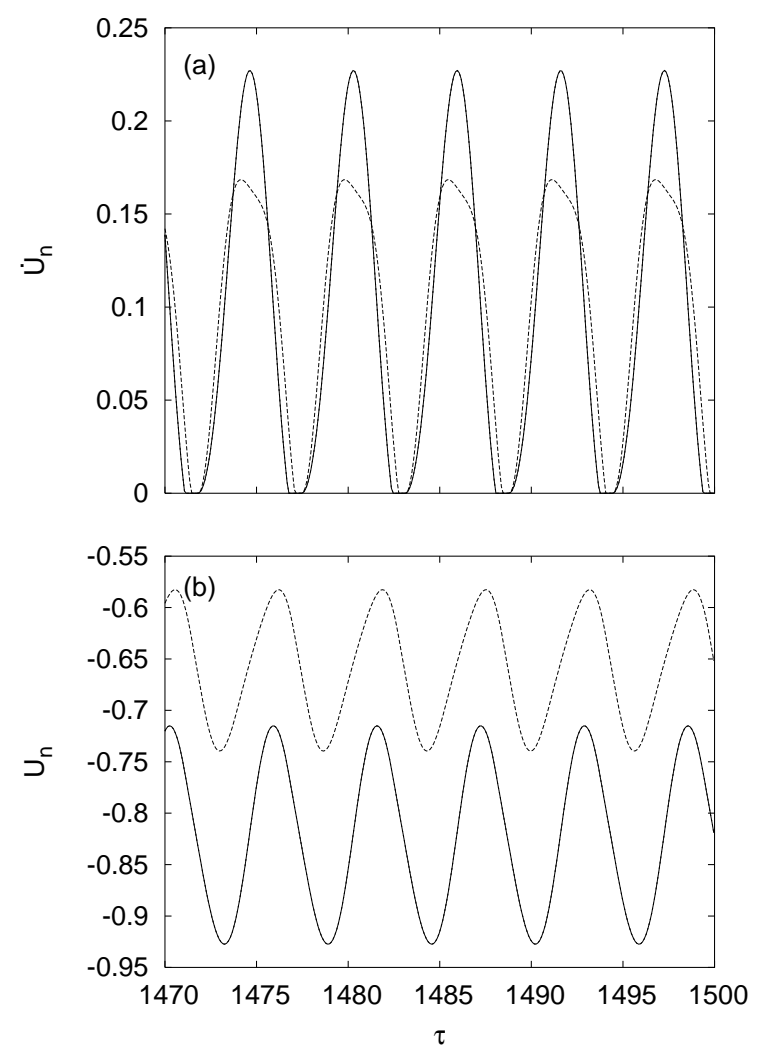

Figure 6: (a) Velocities of blocks as a function of time $\tau$. (b) Positions of blocks as a function of time $t$. The initial position of the first block $U_{1}(\tau=0)=-0.989$. Solid line for blocks 1 and 3, dashed line for block 2 .

\section{Conclusions}

The linearization procedure, described in Section II, does not alter the system behaviour, if the value of the coefficient $a$ is smaller than 0.1 . This means, that the only relevant nonlinearity in this range of $a$ is due to the stick-slip dynamics. Nonlinear character of the velocity dependence of the friction force is not essential for the kind of motion. This result is in accordance with our previous conclusions on the train model of two blocks [19]. From the computational point of view, such a conclusion is optimistic, because the time of calculations is much shorter for a map than for a set of differential equations.

The history-dependent character of the motion below $a=0.1$ can be interpreted as an example of the frequency-locking, which precedes a transition to chaos 20]. When the initial values of the phases of the lateral blocks are close to each other, this frequency-locking leads to the synchronization. If they are oppose, we observe a kind of anti-synchronization, which persists in time. Sim- 

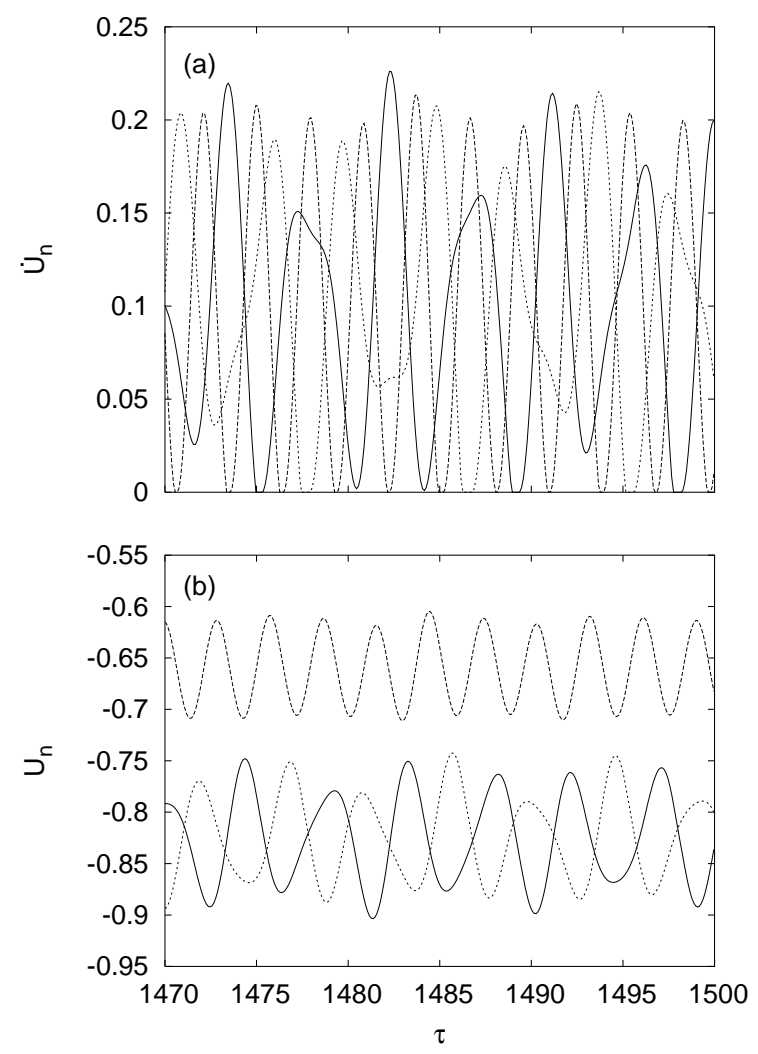

Figure 7: (a) Velocities of blocks as a function of time t. (b) Positions of blocks as a function of time $t$. The initial position of the first block $U_{1}(\tau=0)=+0.989$. Solid line for block 1, dashed line for block 2 and dotted line for block 3 .

ilar effect are observed also in the Hamiltonian ding-a-ling system which can be described by coupled maps [21].

A new question appears, if the frequency-locking could be observed for a system with larger number of blocks, which imitates a real surface. This kind of behaviour is analogous to an excitation in linear systems. What are the consequences for the friction of macroscopic surfaces? At the beginning of this text, we have listed various many-body nonlinear systems. Could these excitations appear in some of this systems for larger number of subsystems? What are the necessary conditions for this kind of motion? To answer these questions, further research is needed, but the effort seems to be profitable. 


\section{Acknowledgments}

The simulations were carried out in ACK-CYFRONET-AGH. The time on SGI 2800 machine is financed by the Polish Committee for Scientific Research (KBN) with grant No. KBN/SGI2800/022/2002.

\section{References}

[1] M. de Sousa Vieira, Phys. Rev. Lett. 82 (1999) 201.

[2] M. de Sousa Vieira, Phys. Lett. A 198 (1995) 407.

[3] H. J. S. Feder and J. Feder, Phys. Rev. Lett. 66 (1991) 2669.

[4] Z. Olami, H. J. S. Feder and K. Christensen, Phys. Rev. Lett. 68 (1992) 1244.

[5] M. Paliy, O. Braun, T. Dauxois and B. Hu, Phys. Rev. E 56 (1997) 4025.

[6] J. Ford, Phys. Rep. 213 (1992) 271.

[7] M. Toda, Prog. Theor. Phys. Suppl. 45 (1970) 174.

[8] T. Prosen and M. Robnik, J. Phys. A 25 (1992) 3449.

[9] G. Casati, J. Ford, F. Vivaldi and W. M. Visscher, Phys. Rev. Lett. 52 (1984) 1861.

[10] R. Burridge and L. Knopoff, Bull. Seismol. Soc. Am. 57 (967) 341.

[11] P. Bak, How Nature Works. The Science of Self-Organized Criticality, Copernicus Springer-Verlag, New York 1996.

[12] B. Gutenberg and C. F. Richter, Ann. Geofis. 9 (1956) 1.

[13] B. N. J. Persson, Sliding Friction: Physical Principles and Applications, Springer, Heidelberg 1998

[14] B. N. J. Persson, Surf. Sci. Rep. 33 (1999) 83.

[15] C. P. Hendriks, PhD Thesis, Technical University Eidhoven, Eidhoven 2000.

[16] J. R. Rice and A. L. Ruina, J. Appl. Mech. 50 (1983) 343.

[17] T. Baumberger, Sol. St. Commun. 102 (1997) 175

[18] L. Bureau, T. Baumberger, C. Caroli and O. Ronsin, C. R. Acad. Sci. Paris, t.2, Série IV, (2001) 699

[19] J. Szkutnik and K. Kułakowski, Int. J. Mod. Phys. C 13 (2002) 41.

[20] E. Ott, Chaos in Dynamical Systems, Cambridge University Press 1993.

[21] P. Gawroński and K. Kułakowski, Comp. Phys. Commun. 147 (2002) 808. 